\title{
Accurate improvement of a mathematical correlation for estimating diffusion coefficient in gaseous hydrocarbons
}

\author{
Mohammad Mohsen Sarafraza,*, Mohammad Jamshidnejada, Ahmad Arabi Shamsabadib, \\ Saeed Lakic and Hadi Ajamib \\ a Chemical Engineering Department, Islamic Azad University, Mahshahr-63519, Iran \\ b Gas Engineering Department, Petroleum University of Technology, Ahwaz-63431, Iran \\ c Chemical Engineering Department, Amir Kabir University, Mahshahr-63516, Iran \\ *Corresponding author at: Chemical Engineering Department, Islamic Azad University, Mahshahr-63519, Iran. Tel.: +98.9166317313; fax: +98.6324230664. \\ E-mail address: mohamadmohsensarafraz@gmail.com (M.M. Sarafraz).
}

\section{ARTICLE INFORMATION}

Received: 30 April 2011

Received in revised form: 19 June 2011

Accepted: 21 June 2011

Online: 31 December 2011

\section{KEYWORDS}

\section{Mathematical correlation}

Diffusion coefficient

Gaseous hydrocarbons

Experimental data

Optimized correlation

Riazi-Whitson correlation

\section{ABSTRACT}

Accuracy of Riazi-Whitson mathematical correlation for estimating the molecular diffusion coefficient in gaseous hydrocarbons has been improved, which decreases the absolute average deviation related to the experimental data using About 486 experimental data points that have been collected from latest existing researches. Likewise, re-optimizing, and statistical calculations have been done to synchronize data to avoid unexpected deviations. As shown in present work, deviation values for results of improved correlation from experimental data are less in compare to Riazi-Whitson original correlation. The absolute average deviations for obtained values of improved correlation are about $9.71 \%$, which is about $14 \%$ for original mentioned correlation. The input parameters are molecular weight, critical properties, and acentric factors of components in the system; mixture molar density; low-pressure gas viscosity and actual viscosity. The last three properties are calculable by proper correlations in chemical handbooks.

\section{Introduction}

Estimation of the diffusion coefficients is an important step in mass-transfer calculations. In the past decades, less attention has been given to predict diffusion coefficients at high pressures. This is mainly because of the difficulty in measuring diffusivity, especially in liquid and gaseous systems, and reported values are not accurate. Gas injection (hydrocarbon, nitrogen, or carbon dioxide) into oil and condensate reservoirs may be attempted to recover more in situ hydrocarbons. In some cases, particularly in naturally fractured reservoirs, it has been a key parameter in estimating the rate and amount of mass transfer by diffusion. The most important property, to obtain such information, is the diffusion coefficient at reservoir conditions. Furthermore, presenting a correlation with less deviation from the real diffusion amount is a need. This investigation provides an improved optimized correlation for estimating the diffusion coefficient near the real states in gaseous hydrocarbons.

In 1970, Dawson et al. [1] investigated on self-diffusion of methane and proposed an embedded reduced parameter correlation which is defined as equation (1):

$$
\frac{\rho D}{\rho D}=a+b \rho_{r}+c \rho_{r}^{2}+d \rho_{r}^{3}+\ldots
$$

Term of $\rho D$ can be obtained from Chapman-Enskog theory [2]. Sometimes later, in 1974, Takahashi [3] modified the Slattery and Bird [4] chart with much more experimental data and presented a better method for estimating the diffusion in gaseous. Two years later, at 1976, Sigmund [5] presented a new precise correlation for binary mixtures of hydrocarbons based on molecular thermodynamic and intermolecular forces, which afterwards, became a reference for Riazi-Whitson correlation. However most of used data was liquid. Admittedly proposed correlation was not proper for estimating diffusion coefficient for gaseous systems. He reported absolute average deviation for liquids about $40 \%$ and for hydrocarbon gaseous about $18 \%$. But in fact, this certainty is not reasonable for real gaseous systems and for some mixtures this deviation percent became more than 27\%. In 1993, Riazi and Whitson [6] proposed a new correlation which surrounded thermo physical properties of the mixtures such as viscosity, density, critical and molecular and energy parameters. The general stem of improved correlation was defined by Riazi as equation (2):

$$
\begin{aligned}
& \frac{\rho D}{\rho D}=a\left(\frac{\mu}{\mu^{\circ}}\right)^{n=b+c P_{r}} \\
& a=1.07, \quad b=-0.27-0.38 \omega, c=-0.05+0.1 \omega, \quad p_{r}=\frac{p}{p_{c}}
\end{aligned}
$$

Wherein: $\omega$ is acentric factor of mixture and can be obtained by linear superimposition of constituent(s) of mixture: $\omega=x_{A} \omega_{A}+x_{B} \omega_{B}$. In Riazi-Whitson correlation, $\rho$ and $\mu$ are mixture molar density and viscosity at the conditions of the system respectively, and $n$ is a weak function of pressure and has a negative value. 


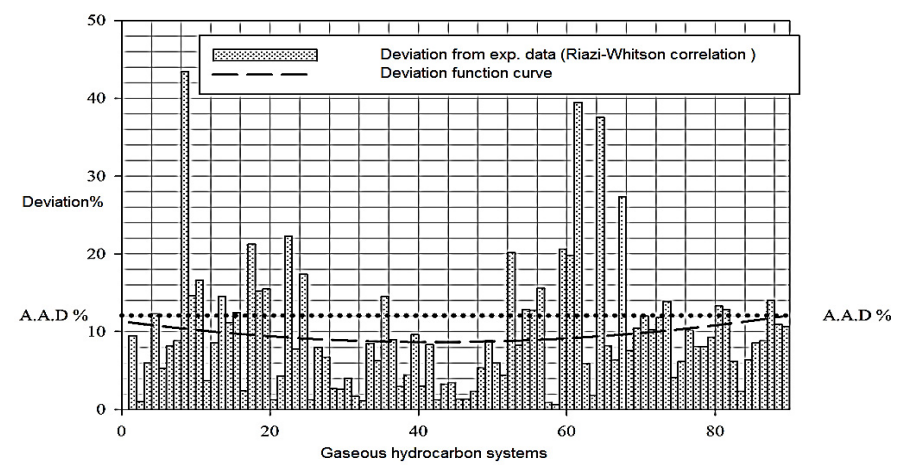

Figure 1. Average deviation of results of Riazi-Whitson (1993) correlation in comparison to experimental data.

The low-pressure density diffusivity product $\rho D$ is calculated from the Chapman- Enskog dilute gas theory correlation [2].

To calculate the, $\dot{\mu}$ Stiel-Thodos correlation [7] has been used which has been presented as equation ( 3 and 4 ):

$$
\begin{aligned}
& \mu_{i}^{0} \cdot \varepsilon_{i}=34 \times 10^{-5} T_{r, i}^{0.94}\left(\text { for } T_{r, i}<1.5\right) \\
& \mu_{i}^{0} \cdot \varepsilon_{i}=17.78 \times 10^{-5}\left[4.85 T_{r, i}-1.67\right]^{\frac{5}{8}} \text { for } T_{r, i}>1.5 \\
& \varepsilon_{i}=\frac{T_{c, i}{ }^{\frac{1}{6}}}{M_{i}^{0.5}\left(0.987 P_{c, i}\right)^{\frac{2}{3}}}
\end{aligned}
$$

For a mixture A and B diluted viscosity $\dot{\mu}$ can be obtained by equation (6):

$$
\mu^{0}=\frac{x_{A} \mu_{A}^{0} M_{A}^{0.5}+x_{B} \mu_{B}^{0} M_{B}^{0.5}}{x_{A} M_{A}^{0.5}+x_{A B} M_{B}^{0.5}}
$$

$\rho D$ is calculable from the following form of the ChapmanEnskog dilute gas theory using the Stiel-Thodos (1962) [8] correlation for estimating the molecular parameters. For a binary $\mathrm{A}$ and $\mathrm{B}$ mixture:

$$
\rho D=\frac{2.2648 \times 10^{-6} T^{0.5} \cdot\left(\frac{1}{M_{A}}+\frac{1}{M_{B}}\right)}{{\sigma_{A B}{ }^{2} \cdot \Omega_{A B}}}
$$

and

$$
\Omega_{A B}=\frac{1.0603}{\mathrm{~T}^{* 0.1561}}+0.193 \times e^{\left(4.475 \mathrm{~T}^{\circ}\right)}+1.7647 \times e^{\left(-89411 . \mathrm{T}^{*}\right)}+1.03587 \times e^{\left(-1.52996 . \mathrm{T}^{\circ}\right)}
$$

Note that: $\mathrm{T}^{*}=\frac{T}{\varepsilon_{A B}}, \varepsilon_{A B}=\left(\varepsilon_{A} \cdot \varepsilon_{B}\right)^{0.5}, \varepsilon_{i}=65.3 T_{c, i} Z_{c, i}{ }^{18 / 5}$.

$\sigma_{A B}=\frac{\sigma_{A}+\sigma_{B}}{2}$ $\sigma_{i}=1.866 \cdot V_{c, i}^{\frac{1}{3}} \cdot Z_{c, i}^{-\frac{6}{5}}$

Chapman-Enskog correlation was developed with limited experimental data bank and for the better adaption; this correlation should be re-constructed using new experimental data.

\section{Results and discussion}

It can be noted that Chapman-Enskog correlation was developed in 1962 with limited experimental data bank. Also this correlation cannot provide reasonable results for wide range of substances. However it becomes most common used correlation among the engineers. Another important point that must not be neglected implies that Riazi and Whitson used the latest available experimental data, however many experimental investigations have been done since that time which include the worth and exact experimental data. These data collections are usable for re-optimizing and re-constructing the existed correlations to improve their accuracy. In addition, statistical calculations show that omitting some hydrocarbon systems (for instance at high-pressure and supercritical conditions), leads to more accuracy correlation In this research general supposition of Riazi-Whitson has been reconstructed by more than 200 latest data points which were not considered in Riazi-Whitson proposal correlation. Accordingly exponent terms of assumption as well as constant coefficients, have been change to optimal values which is shown as equation (11 to 13 ):

$$
\begin{aligned}
& a=1.086 \\
& b=-0.27-0.38 \times \omega^{0.94} \\
& c=-0.04887+0.1 \times \omega^{0.91}
\end{aligned}
$$

Repetition of reconstruction process for Chapman-Enskog intermolecular term $\Omega_{A B}$ re-develops the accurate correlation which is shown as equation (14) and should be replaced by the old ones:

$$
\Omega_{A B}=0.7158+1.9905 \times \mathrm{e}^{\left(-3.6276 \times T^{\circ}\right)}+1.1969 \times \mathrm{e}^{\left(-1.3502 \times T^{*}\right)}+0.47079 \times \mathrm{e}^{\left(-0.2639 \times \times T^{\circ}\right)}
$$

Figure 1 typically represents the obtained results for RiaziWhitson correlation without optimizing process. Rectangle bars with hachures shows the deviation values in compare to experimental data. Also, after data synchronization, Data analysis shows that absolute average deviation (A.A.D. \%) of Riazi-Whitson for about 100 new and earlier experimental data 
Table 1. Data bank references for gaseous hydrocarbon diffusion coefficients.

\begin{tabular}{lllll}
\hline Group & Number of data point & Pressure range & Temperature range & References \\
\hline $\mathbf{C}_{\mathbf{1}}-\mathbf{C}_{\mathbf{3}}$ & 44 & $14-207$ & $311-378$ & {$[5]$} \\
C1- $\mathbf{C} 4$ & 58 & $14-174$ & $311-378$ & {$[513-367$} \\
N2-C1 & 28 & $35-138$ & $313-351$ & {$[5]$} \\
C1-C2 & 30 & $69-275$ & $155-354$ & {$[14]$} \\
C1-C1 & 16 & $10-416$ & 313 & {$[1]$} \\
N2-C2 & 30 & $7-173$ & $155-414$ & {$[1]$} \\
C1-C3/C1-C5 & $55 / 85$ & $20-143$ & Not mentioned exactly & {$[20]$} \\
Dissipated data points & 138 & $6-221$ & & {$[13,16,18]$} \\
\hline
\end{tabular}

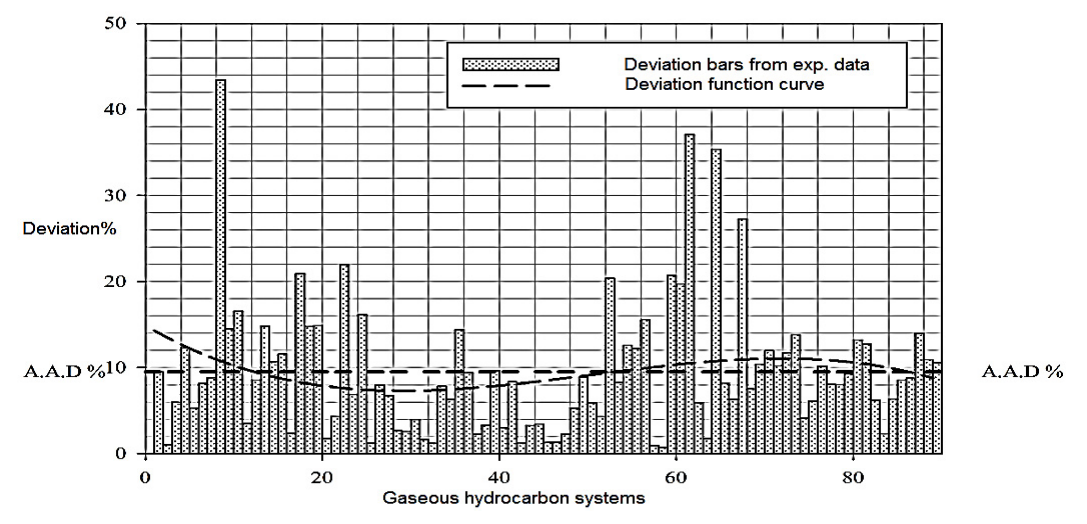

Figure 2. Average deviation of modified correlation of Riazi-Whitson (this work).

point is about $14 \%$. however this average after improving has been reduced to about $9.71 \%$. Deviation function curve, as shown in Figure 1 and 2, mathematically express the order of deviation. This value is 2 for Riazi-Whitson and is 3 for this work, respectively. Higher values of deviation function order result in, accurate estimated values.

As shown in two previous figures, a rough comparison between deviation curve of Riazi-Whitson and improved correlation implies on this fact that error order of improved correlation is higher than Riazi-Whitson correlation which is obviously confirms that modified version provides more reasonable values in compare to Riazi-Whitson original correlation. Table 1 provides a supplementary data including the reference for experimental data, which have been used for optimizing in presented work. It is noticed that there are some other data points which, were dissipated and there were no regular situation to bring them into following table.

For better Comparison, Figure 3 has been plotted which shows the better conformation of improved correlation for about 75 gaseous experimental data points in compare to RiaziWhitson correlation.

Figures 4 and 5 typically represent the accuracy of results of this work schematically in compare to experimental data and confirm that experimental data have had a less deviation in related to optimized correlation in compare to Riazi-Whitson method.

Also the estimated values using improved correlation in comparison to experimental data and obtained values from original Riazi-Whitson original correlation is given in Figure 6.

\section{Conclusion}

In this contribution, collection of latest experimental data related to measuring the diffusion coefficients in gaseous hydrocarbon systems has been gathered. After smoothing and synchronization of the data, statistical calculations have been done. Duplicate data has been eliminated. Chapman-Enskog correlation has been reconstructed by updated experimental data and assumption of Riazi-Whitson correlation with constant coefficient and exponent has been change to optimum

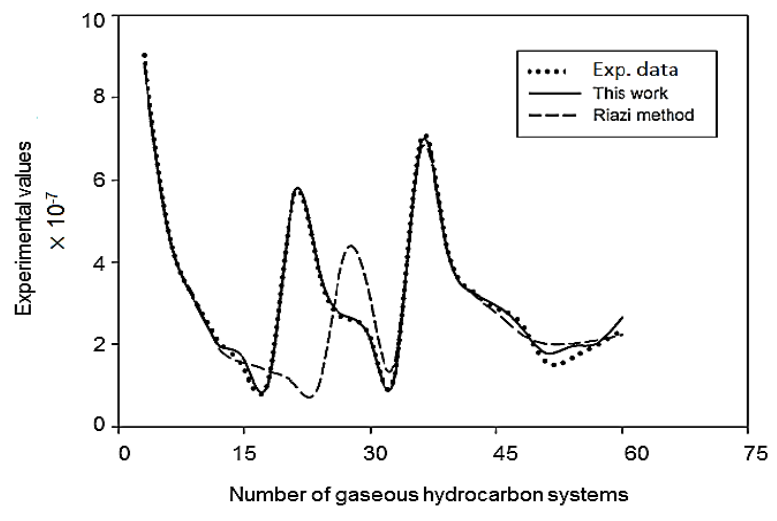

Figure 3. Improved correlation results in comparison to Riazi-Whitson correlation values.

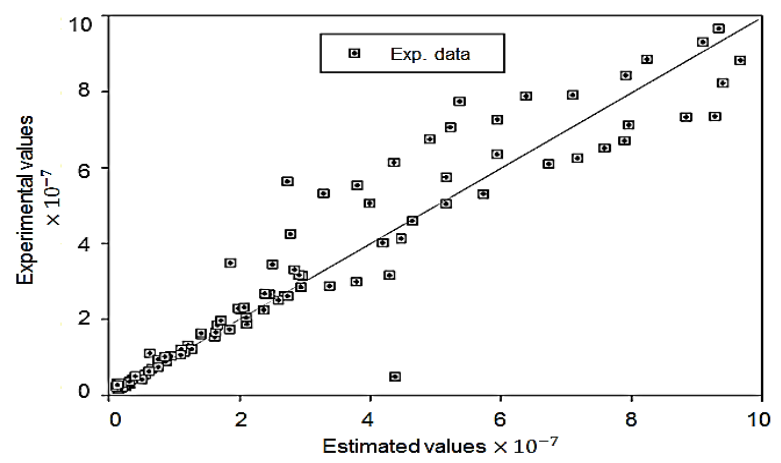

Figure 4. Accuracy of obtained results for Riazi-Whitson in comparison to experimental data. 
situation, which provides A.A.D \% about $9.71 \%$ for gaseous hydrocarbon systems. This value in comparison to $14 \%$ claimed by Riazi-Whitson correlation is reasonable and acceptable accuracy. New improved correlation is recommended for estimating the diffusion coefficient in gaseous hydrocarbons at low, moderate and even high pressure circumstances.

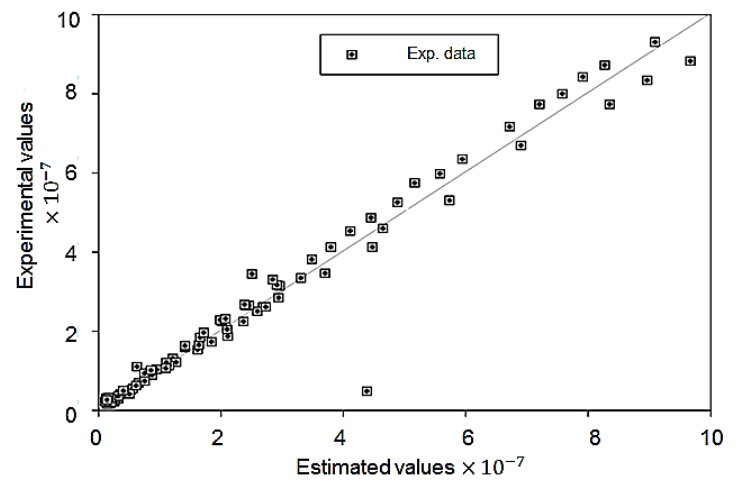

Figure 5. Accuracy of obtained results for Riazi-Whitson in comparison to experimental data.

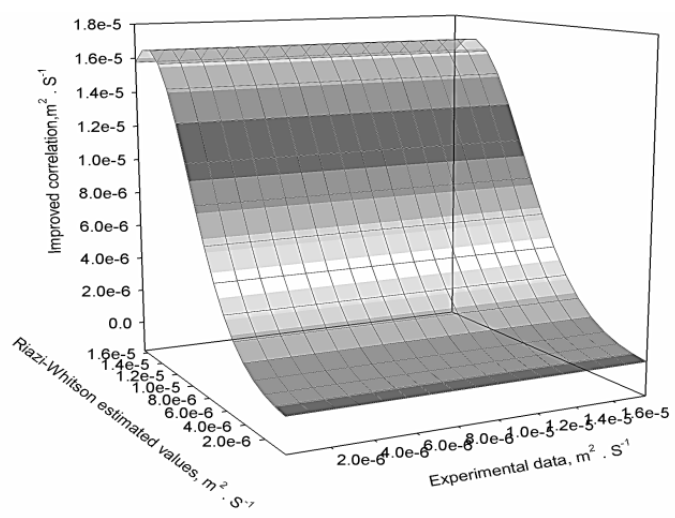

Figure 6. Comparison between Experimental data and Re-optimized correlation results and original Riazi-Whitson values.

\section{Acknowledgements}

Authors of this article wish to appreciate Islamic Azad University, branch of Mahshahr for their financial supports. Also they appreciate to Dr. Mohammad Jamshidnejad for his scientific supports.

\section{Nomenclatures}

a, b, c, d: Constant parameter D: Diffusion coefficient $\left[\mathrm{m}^{2} . \mathrm{S}^{-1}\right]$ $\mathrm{M}_{\mathrm{A}}$ : Molecular weight of solute $\mathrm{M}_{\mathrm{B}}$ : Molecular weight of solvent n: Exponent number

P: Pressure [bar]

$P_{c}$ : Critical pressure [bar]

$\mathrm{P}_{\mathrm{r}}$ : Reduced pressure

T: Temperature $[\mathrm{K}]$

$\mathrm{T}_{\mathrm{c}, \mathrm{i}}$ : Critical temperature [K]

$\mathrm{T}_{\mathrm{r}, \mathrm{i}}$ : Reduced temperature

$\mathrm{T}^{*}$ : Specific temperature, see correlation 8

$\mathrm{V}_{\mathrm{ci}}$ : Critical volume $\left[\mathrm{m}^{3}\right]$

$\mathrm{Z}_{\mathrm{C}, \mathrm{i}}$ Compressibility factor

$\Omega_{\mathrm{AB}}$ : Molecular parameter see section

$\mu$ : Viscosity [N.m $\left.{ }^{-1}\right]$

$\mu^{0}$ : Diluted viscosity [N.m-1 $\left.{ }^{-1}\right]$

$\varepsilon_{\mathrm{AB}}$ : Lennard-jones parameter $\varepsilon_{\mathrm{i}}$ : See discussion

$\omega:$ Acentric factor

$\sigma_{\mathrm{i}}$ : See introduction section iii

$\rho:$ Density $\left[\mathrm{Kg}^{-\mathrm{m}^{-3}}\right]$

$\rho^{\circ}$ : Diluted density $\left[\mathrm{Kg}^{-\mathrm{m}^{-3}}\right]$

\section{References}

[1]. Dawson, R.; Khoury, F.; Kobayashi, R. Aiche. J. 1970, 16, 725-729.

[2]. American Petroleum Institute Technical Data Book-Petroleum Refining, $3^{\text {th }}$ edition, API Washington D.C., 1977.

[3]. Takahashi, S. J. Chem. Eng. Japan 1974, 7, 417-420.

[4]. Slattery, J. C.; Bird, R. B. Aiche. J. 1961, 7, 611-616.

[5]. Sigmund, P. M.; Can. J. Pet. Technol. 1976, April-June, 48-57.

[6]. Riazi, R.; Whitson, C. H. Ind. Eng. Chem. Res. 1993, 32, 3081-3088.

[7]. Stiel, L.; Thodos, G. Aiche J. 1958, 4, 137-142.

[8]. Stiel, L. I.; Thodos, G. J. Chem. Eng. Data 1962, 7, 234-236.

[9]. Graue, D. J. A Numerical Method for Smoothing Thermodynamic Data, Ph.D. Dissertation, California Institute of Technology, 1965.

[10]. Reamer, H. H.; Opfell, J. B.; Sage, B. H. Ind. Eng. Chem. 1956, 423, 275 282.

[11]. Riazi, M. R.; Daubert, T. E. Aiche J. 1980, 26, 386-390.

[12]. Riazi, M. R.; Daubert, T. E. Ind. Eng. Chem. 1987, 26, 755-759.

[13]. Berry, V. J.; Koeller, R. C. Aiche J. 1960, 6, 274-279.

[14]. Bird, R. B.; Stewart, W. E.; Lightfoot, E. N. Transport Phenomena, John Wiley \& Sons, New York, 1960.

[15]. Chung, T. H. M; Ajlan, M; Lee, L. L; Starling, K. E. Eng. Chem. Res. 1988 27, 671-679.

[16]. Mathur, G. P.; Thodos, G. Aiche. J. 1965, 11, 613-616

[17]. Prausnitz, J. M.; Cheue, P. L. Computer Calculations of High-Pressure Vapor-Liquid Equilibria, Prentice-Hall, Englewood Cliffs, NJ, 1968.

[18]. Poling, B. E.; Prausnitz, J. M.; O' Connell, J. P. The properties of gases and liquids, $5^{\text {th }}$ Edn, McGraw-Hill, New York, 2000. 